\title{
Establishment of HLA class I and MICA/B null HEK- 293T panel expressing single MICA alleles to detect anti-MICA antibodies
}

Ji-Ho Jeon

Catholic University of Korea

In-Cheol Baek

Catholic University of Korea

Cheol-Hwa Hong

Catholic University of Korea

Ki Hyun Park

Catholic University of Korea

Hyeyoung Lee

Catholic University of Korea

Eun-Jee Oh

Catholic University of Korea

Tai-Gyu Kim ( $\sim$ kimtg@catholic.ac.kr)

Catholic University of Korea

\section{Research Article}

Keywords: anti-MICA antibody detection, transplant, antigens

Posted Date: May 3rd, 2021

DOI: https://doi.org/10.21203/rs.3.rs-442120/v1

License: (c) (7) This work is licensed under a Creative Commons Attribution 4.0 International License. Read Full License

Version of Record: A version of this preprint was published at Scientific Reports on August 3rd, 2021. See the published version at https://doi.org/10.1038/s41598-021-95058-8. 


\section{Abstract}

Pre- and post-transplantation anti-MICA antibody detection development are associated with an increased rejection risk and low graft survival. We previously generated HLA class I null HEK-293T using CRISPR/Cas9, while MICA and MICB genes were removed in this study. A panel of 11 cell lines expressing single MICA alleles was established. Anti-MICA antibody in the sera of kidney transplant patients was determined using FCM (flow cytometric method) and the Luminex method. In the 44 positive sera, the maximum FCM value was 2,879 MFI compared to $28,135 \mathrm{MFI}$ of Luminex method. Eleven sera (25\%) were determined as positive by FCM and 32 sera (72\%) were positive by the Luminex method. The sum of total MICA antigens, MICA $* 002, * 004, * 009$, *019, and $\star 027$ correlation showed a statistically significant between the two methods $(P=0.0412, P=0.0476, P=0.0019, P$ $=0.0098, P=0.0467$, and $P=0.0049)$. These results demonstrated that HEK-293T-based engineered cell lines expressing single MICA alleles were suitable for measuring specific antibodies against MICA antigens in the sera of transplant patients. Studies of antibodies to MICA antigens may help to understand responses in vivo and increase clinical relevance at the cellular level such as complement-dependent cytotoxicity.

\section{Introduction}

The major histocompatibility complex $(\mathrm{MHC})$ region, which plays an important role in protecting against pathogens, includes both polymorphic and multi-copy genes ${ }^{1}$. The MHC class I chain-related gene A (MICA), which is located on human chromosome 6 , approximately $46.4 \mathrm{~Kb}$ centromeric to the HLA-B locus, encodes a protein with three extracellular domains, a transmembrane (TM) segment, and a carboxy-terminal cytoplasmic tail 2,3. MICA polymorphisms have been identified in over 220 alleles in the IPD-IMGT/HLA database release 3.43.0 (http://www.ebi.ac.uk/imgt/hla). MICA is found in many cell lines as well as in primary cells such as endothelial cells, fibroblasts, and gastrointestinal epithelial cells, and cell stress can induce its expression ${ }^{4}$. MIC proteins are stress markers for cells, because the expression of these molecules is induced by heat, viral infection, inflammation, and DNA damage ${ }^{5-9}$. The extracellular domains consist of $a 1, a 2$, and a3 chains that flexibly

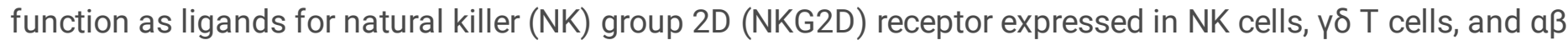
CD8 T cells ${ }^{10}$. It is also expressed in several epithelial tumors, including lung, breast, kidney, ovarian, prostate, and colon carcinomas ${ }^{11}$.

The pretransplant crossmatch, which involves testing the recipient's serum for cytotoxicity against the donor cells (lymphocytes), was introduced in the early days of renal transplantation ${ }^{12}$. In recent years, there have been reports that human leukocyte antigen (HLA) antibodies can be associated with allograft loss ${ }^{13,14}$. Several techniques are currently used to detect anti-HLA antibodies to decrease the rate of organ rejection and improve survival, including cell-based methods such as complement-dependent cytotoxicity (CDC), flow cytometric method (FCM), and solid-phase-based methods such as enzyme-linked immunosorbent assay (ELISA), and Luminex.

In addition to anti-HLA antibodies, other antibodies against endothelial cells have been found to have important clinical significance in transplantation ${ }^{15-17}$. Pre-transplantation detection of anti-MICA antibodies is associated with an increased risk of rejection and low graft survival ${ }^{18}$. Furthermore, post-transplantation anti-MICA antibody development is associated with a great rate of rejection and graft failure ${ }^{12,19}$. The most popular method for antibody identification with single antigen beads is based on fluorescent beads using Luminex technology, but 
many technical and interpretation issues remain unclear ${ }^{20,21}$. The MICA molecule expressed in cells is considered to reflect the situation in the human body in terms of structural and expression levels compared to the recombinant protein bound to the bead. In a previous study, single MICA alleles were expressed through gene transfer in C1R cell lines or High Five insect cell lines that did not express HLA to study anti-MICA alloantibodies at the cell level ${ }^{22}$.

We previously generated the HEK-293T cell line from which the HLA class I gene was removed using CRISPR/Cas9 ${ }^{23}$. In this study, we generated the cell line from which MICA and MICB genes were removed. Based on this cell line, a panel of cell lines that expresses 11 types of MICA alleles frequent in Koreans was established. The presence of anti-MICA alloantibodies in the sera of kidney transplant patients was determined using this panel of cell lines expressing single MICA alleles and compared to the results obtained using the Luminex method.

\section{Results}

\section{Establishment of MICA cell lines expressing single MICA antigen}

We selected the H1E-25 HEK-293T cell line from which HLA class I was deleted to rule out alloantibody reactions against HLA ${ }^{23}$. Three plasmids encoding the Cas9 protein and gRNA to target MICA exon 2 (MAE2), MICB exon 2 (MBE2), and MICA/B exon 3 (MABE3) were used for complete deletion of the MICA/B molecule (Fig. 1A). These three plasmids were co-transfected with H1E-25 HEK-293T cells. Cells that were successfully transfected and expressed fluorescence were separated into single cells using fluorescence-assisted cell sorting (FACS) and cultured into 96-well plates. For the 48 clones growing, the cell lines with predicted deletions were investigated via PCR of the regions containing exon 2 and exon 3 (Fig. 1B). There were 12 heterozygous and six homozygous detectable changes (DCPs) in MICA and 14 heterozygous and 13 homozygous DCPs in MICB. We found that exon 2-3 of MICA/B was completely deleted in clones H1ME-5, -15, and -26 (Fig. 1C). MICA was cut at the predicted cut sites, and 267 bp were deleted from all three clones. However, in the exon 3 site of MICB, a total of 265 bp was deleted from all three clones by cutting $24 \mathrm{bp}$ ahead of the predicted cut site.

Although MICA and MICB were weakly expressed in the H1E-25 HEK-293T cell line, it was confirmed that they were not expressed at all in the three clones from which both MICA and MICB were removed. In the subsequent experiments, the selected H1ME-5 HEK-293T cell line was used (Fig. 2A). To establish a cell line panel expressing single MICA alleles, H1ME-5 HEK-293T cells were transduced with lentiviruses encoding 11 MICA alleles, MICA*002, *004, *007, *. 008, *009, *010, *011, *012, *019, *027, and *049. MICA-positive cells were sorted and cultured on day 6 after transduction. FACS analysis confirmed the high expression of 11 single MICA alleles (Fig. 1B). To investigate whether these cell panels were suitable for measuring anti-MICA antibodies in the patient serum, the serum determined as positive and the serum determined as negative were subjected to flow cytometric method (FCM) after fluorescence staining (Fig. 2C). Negative serum (13,101 MFI) was not measured in all cells expressing a single MICA allele in the same manner as H1ME-5, which did not express MICA. Positive serum $(17,815 \mathrm{MFI})$ responded only to certain MICA alleles such as MICA*002, $* 011$, and $* 049$. These results indicate that the non-specific response of serum in the cell panel based on H1ME-5 HEK-293T cells is very low.

\section{Responses of FCM and Luminex method to MICA alleles in patient serum}


In this study, 11 MICA antigens used for FCM were selected to be present at a frequency of $2 \%$ or more in Koreans (Table 1). A total of 15 MICA antigens were included in the Luminex method, but six antigens were not reported in Koreans, and two antigens had a frequency of less than $1 \%$. Seven antigens were detected by both the FCM and Luminex methods. Because MICA*008 and $* 027$ or $* 009$ and $* 049$ alleles showed the same amino acid sequences of the extracellular domain on the cell surface, the Luminex method detected only MICA*027 and *009, while FCM identified all these antigens.

The 64 sera collected from kidney transplant patients used in this study consisted of 44 positive sera and 20 negative sera using the Luminex screening kit (Fig. 3). The cut-off value for the FCM test was calculated as mean +4 SD based on the data on the allele antigen with the largest deviation from 20 negative sera and determined as $237 \mathrm{MFI}($ mean $=59, \mathrm{SD}=45)$. Similarly, the cut-off value for the Luminex method was determined to be 1,247 $\mathrm{MFI}$ (mean $=283, \mathrm{SD}=241)$. Among the negative sera, the maximum values for FCM and Luminex methods were 209 and $822 \mathrm{MFI}$, respectively.

After testing the positive 44 sera using the FCM and Luminex methods, the results from FCM were sorted from the highest value based on the allele with the highest response in each serum, and the results from the Luminex method were listed corresponding to FCM (Fig. 3). The maximum value of FCM was 2,879 MFI, which was approximately $1 / 10$ of the $28,135 \mathrm{MFI}$ of the Luminex method. Eleven sera with more than this cut-off value were determined to be positive by FCM. Based on the above cut-off value, there were $11(25 \%)$ and 32 (72\%) positive serum samples by FCM and Luminex method, respectively.

\section{Distribution of positive responses according to individual MICA antigens}

In the analysis of responses on individual MICA antigens (Fig. 4), the order of MICA antigens that showed strong responses (mean) in the FCM test was *009 (179 MFI), *012 (169 MFI), *007 (147 MFI), *002 (143 MFI), *027 (133 $\mathrm{MFI}), * 008$ (124 MFI), *004 (115 MFI of), *019 (115 MFI), *049 (79 MFI), *010 (78 MFI), and *011 (45 MFI). The order of MICA antigens in the Luminex method was *002 (4,477 MFI), *005 (4,333 MFI), *028 (4,209 MFI), *007 $(4,190 \mathrm{MFI}), * 009(4,044 \mathrm{MFI}), * 019(3,929 \mathrm{MFI}), * 027$ (3,920 MFI), *006 (3,849 MFI), *004 (3,623 MFI), *001 (3,427 $\mathrm{MFI}), * 015(3,409 \mathrm{MFI}), * 046(2,957 \mathrm{MFI}), * 017(2,925 \mathrm{MFI}), * 012(2,199 \mathrm{MFI})$, and *018 (1,858 MFI).

\section{Correlation between the results of FCM and Luminex method}

The correlation between the results of the two assays was analyzed in 11 serum samples that were positive in the FCM assay (Fig. 5). First, the total response in each serum sample was compared for each of the seven individual antigens present in both assays. MICA ${ }^{\star 002}, * 004, * 009, * 019$, and $* 027$ correlation showed a statistically significant $(P=0.0412, P=0.0476, P=0.0019, P=0.0098, P=0.0467$, and $P=0.0049$, respectively $)$. However, the MICA*007 and *012 alleles showed no significant correlation.

\section{Response patterns to MICA alleles by FCM}

When analyzing the response pattern to each antigen for 11 positive sera in the FCM test, six sera were positive for more than five antigens, three were positive for two antigens, and three were positive for a single antigen. Two sera positive for a single antigen showed specific reactions to MICA $* 007$ and $* 049$, respectively. Two of the three sera positive for the two antigens reacted equally to MICA $* 002$ and $* 011$. In the sera positive for eight antigens, two sera responded identically to MICA*004, *007, *008, *009, *010, *012, and *027. MICA*008 and *027, which had the same ECD amino acid sequence, showed the same reaction (Fig. 5). However, MICA*009 and *049, which 
have the same ECD amino acid sequence, did not show the same reaction. The reactions to MICA*010 and *011, which existed in Koreans but were not included in the Luminex method, were $36 \%$ and $27 \%$, respectively.

\section{Discussion}

In the initial study on allogeneic antibodies against MICA antigens, a cell line expressing a single MICA antigen was generated using m-HMy2. The C1R cell line weakly expresses the HLA-C gene but does not express HLA-A, HLA-B, or MICA. It was used for the detection of antibodies against MICA antigen reaction through CDC assay or for identification of shared epitopes by antibody absorption in patient sera ${ }^{24,25}$. The K562 cell line, which does not express HLA, expresses a single HLA antigen and is used for FCM or CDC tests. However, MICA was expressed and was not used for studies on a single MICA antigen ${ }^{26}$. High Five insect cells that do not express human alloantigens were also used to generate single MICA-expressing cell lines, but the recombinant glycoproteins on the cell surface were found to cause cross-reactions with antibodies in human serum ${ }^{22}$. As it was not possible to obtain a cell line expressing a single MICA antigen, recombinant soluble proteins with extracellular domains of each MICA antigen produced in Escherichia coli were mainly used for ELISA or Luminex method from the beginning ${ }^{18,27}$. We previously used the CRISPR/Cas9 to remove all the HLA-A, -B, and -C genes of the HEK-293T cell line to establish a cell line without allogeneic response to HLA ${ }^{23}$. In this study, MICA and MICB genes were removed to establish a cell line without both HLA and MICA/B genes (Fig. 2). This cell line confirmed that nonspecific reactions did not occur in either the positive or negative sera of all patients and was used as internal negative control in this study.

As DNA typing methods for MICA have been developed precisely, more MICA alleles have been reported worldwide, and the distribution of the MICA allele in Koreans has also been well investigated ${ }^{28}$. In this study, 11 types of MICA alleles with a frequency of $2 \%$ or more in Koreans were selected to generate cell lines expressing a single MICA antigen for FCM. Compared to the currently clinically used Luminex method, antibody responses to MICA*010 and *011 antigens, which were not included in the Luminex method, were found to be $36 \%$ and $27 \%$, respectively (Table 2). MICA*001, *005, *015, *018, *028, and ${ }^{*} 046$ antigens included in the Luminex method were not present in Koreans, but reactive antibodies were present (Fig. 4). This phenomenon is known to be due to cross-reactions between MICA antigens that share common epitopes ${ }^{25}$. For example, an antibody against MICA*001 cross-reacts with MICA*001, *012: 01, *018: 01, *007: 01, *002: 01, and *017 alleles belonging to antigen group 1, which shares the same epitope. In FCM, six out of 11 antibodies in the positive sera showed strong cross-reactivity against five or more antigens. There was no significant correlation between *007 and *012 alleles in the serum correlation study for the antigens detected by FCM and Luminex method (Fig. 4). Since MICA*008 and *027 alleles had the same extracellular domain, *008 was not included in the Luminex method, but antibody responses to both antigens were measured by FCM as expected (Table 2). However, MICA*009 and *049 alleles differ in one amino acid sequence in the intracytoplasmic region in exon 6 , and the antibody responses are inconsistent, even though the extracellular domains are identical. These results may be due to the fact that MICA antigens are expressed on the cell surface in FCM, but, in the Luminex method, they are recombinant soluble proteins attached to beads. Previous studies on anti-HLA antibody suggest that conformational changes can occur due to the attachment of recombinant soluble HLA molecules to the beads ${ }^{26}$.

Initially, in the field of organ transplantation, tests to measure antibodies for histocompatibility mainly used the CDC assay and FCM, but the sensitivity increased dramatically as solid phase assays such as ELISA or Luminex 
method were developed. These sensitive tests can increase the early detection of patients at risk for transplant rejection, but low levels of antibodies may not be relevant in clinical events. In this study, the Luminex test was more sensitive than the FCM test, and only few serum positives by the Luminex method were found to be positive by FCM (Fig. 3). All 11 serum samples positive by FCM showed more than 10,000 MFI by the Luminex method. Pre-transplant donor-specific HLA antibodies measured by both the CDC and Luminex methods in Korean kidney transplant patients were identified as risk factors for microvascular inflammation in allograft biopsy, but antiMICA antibodies measured using the Luminex method did not reach statistical significance ${ }^{29}$. In a study on prevalence of MICA antibodies in renal biopsies after transplantation, the MFI value was significantly higher in patients with interstitial fibrosis and tubular atrophy (IFTA) II or III ( $\mathrm{n}=3$, median \pm SE, $21919.0 \pm 2581.0)$ than in patients without IFTA II or III $(n=9$, median $\pm S E, 500.0 \pm 155.8)(P=0.009){ }^{30}$.

The results of this study confirmed that the HEK-293T cell line, in which both HLA and MICA/B genes were removed using CRISPR/Cas9 technology, was suitable for measuring antibodies against single MICA antigens in the serum of transplant patients. In FCM, antibodies were tested only for lgG, but simultaneous measurement of IgM and other immunoglobulins and other subclass antibodies may be required. Studies on antibodies against MICA antigens at the cellular level such as CDC and antibody-dependent cytotoxicity in NK cells may help to better understand responses in vivo and increase clinical relevance. Following this study, studies to measure antibodies against MICB antigens are being conducted, and it is expected that the same approach may be applied on various non-HLA antigens in the future.

\section{Methods}

\section{Cell culture}

MICA alleles of peripheral blood mononuclear cells (PBMCs) were selected using SBT at the Catholic Hematopoietic Stem Cell Bank. All cell lines HEK-293T (CRL-3216; ATCC, Manassas, VA, USA), HLA class I null293T (H1E-25), HLA class I, and MICA/B null-293T (H1ME-5) were cultured in Dulbecco's modified Eagle's medium (Lonza) supplemented with 10\% fetal bovine serum (Hyclone, Logan, UT, USA), 1\% L-glutamine (Lonza), and 1\% penicillin-streptomycin (Lonza).

\section{Sera of kidney transplant patients}

A total of 64 kidney transplant samples were provided by the Department of Laboratory Medicine (The Catholic University of Korea, Seoul, Korea). Samples were marked with serial numbers and stored at $-20^{\circ} \mathrm{C}$. When used in the experiment, they were thawed at $25^{\circ} \mathrm{C}$ and vortexed. All subjects provided informed consent to participate in the study. Also, written informed consent was obtained from each participant and their parents or guardians. This study protocol was approved by the Institutional Review Board (IRB) of the Catholic University of Korea (IRB Number: MC13SISI0126 [DNA], MC19SNSI0068 [serum]), Seoul, Korea, and conducted in accordance with the Declaration of Helsinki. First, the presence of MICA in the sera was analyzed using LABScreen ${ }^{\text {TM }}$ Mixed (One Lambda Inc, Canoga Park, CA, USA).

\section{Deletion of both MICA and MICB genes using CRISPR/Cas9}

Three guide RNAs (gRNAs) were used targeting MICA exon 2 (MAE2): 5'-GGCAAAGCCCCAGGGACAGTGGG-3'; MICB exon 2 (MBE2): 5'-GCTATGACAGGCAGAAACGCAGG-3'; MICA/B exon 3 (MABE3): 5'- 
GTCCTCCAGAGCTCAGACCTTGG-3'. All-in-one plasmids, including both gRNA and Cas9 genes, were obtained from Genscript (PX458 and PX459). H1E-25 cells were seeded at $2 \times 10^{6}$ cells/10 mL in antibiotic-free Dulbecco's modified Eagle's medium (Lonza). Twenty-four hours later, a mixture of individual all-in-one plasmids specific for each of the targets was transferred to the H1E-25 cell line using Lipofectamine reagent (Invitrogen, Carlsbad, CA, USA). At $48 \mathrm{~h}$ after transfection, the cells were analyzed using flow cytometry. Six days after transfection, cells negative for MICA/B were sorted, and clones were established. To establish the MICA/B-null cell line, cells cotransfected with three plasmids were harvested in autoMACS Rinsing Solution (Miltenyi Biotec, Bergisch Gladbach, Germany) and stained with anti-MICA-PE (FAB1300P; R\&D Systems, Minneapolis, MN, USA) and antiMICB-APC (FAB1599A; R\&D Systems) for 30 min at $4^{\circ} \mathrm{C}$. Live, GFP-negative, and MICA/B-negative 293T cells were sorted, and single cells were seeded in 96-well plates using MoFlo XDP cell sorters (Beckman). Positive cells were sorted, and single cells were seeded onto 96-well plates using MoFlo XDP cell sorter. At 2-3 weeks after sorting, single-cell clones were established.

\section{PCR and sequencing for detection of deleted MICA and MICB genes}

Two-three weeks after sorting, MICA/B-negative single-cell clones were established and cultured on 6-well plates $(n=188)$. Clonal genomic DNA was isolated from $1 \times 10^{5}-10^{6}$ cells from each clone using a TIANamp Genomic DNA kit (Tiangen Biotech, Beijing, China) according to the manufacturer's instructions. Amplification of each target region was carried out using PCR with forward and reverse primers: MICA, 5'-

CGTTCTTGTCCCTTTGCCCGTGTGC-3' (forward) and 5'-GAATTGGAGGGAGAGGAGAGC-3' (reverse); MICB, 5'AGCCCCACAGTCTTCGTTAC-3' (forward) and 5'-CCAGGGTCGGTACCTGTTCT-3' (reverse). The PCR program consisted of one cycle of $95^{\circ} \mathrm{C}$ for $3 \mathrm{~min}, 35$ cycles of $98^{\circ} \mathrm{C}$ for $20 \mathrm{~s}, 65^{\circ} \mathrm{C}$ for $15 \mathrm{~s}$, and $72^{\circ} \mathrm{C}$ for $20 \mathrm{~s}$; one cycle of $72^{\circ} \mathrm{C}$ for $2 \mathrm{~min}$; and one cycle of $10^{\circ} \mathrm{C}$ for $5 \mathrm{~min}$. PCR products were analyzed by Gel Doc XR+ system (Bio-Rad, Hercules, CA, USA) on 2\% agarose gels, using SYBR Green and a 100-bp ladder (Bioneer, Daejeon, Korea) for screening of predicted large deletion clones. PCR products of selected clones were analyzed by Sanger sequencing (Cosmo Genetech, Seoul, Korea) using the same primers.

\section{Production of lentivirus expressing single MICA alleles}

Commercially available kits were tested for PBMC RNA isolation using NucleoSpin ${ }^{\circledR}$ RNA (Macherey-Nagel, GmbH, Duren, Germany), according to manufacturer protocols were followed for the kit. cDNA was synthesized using the SuperiorScript III cDNA Synthesis kit (Enzynomics, Daejeon, Korea). Amplification of each MICA allele was carried out using PCR with one forward and two reverse primers: 5'-ATGGGGCTGGGCCCGGTCTT-3' (forward), 5'CTAGGCGCCCTCAGTGGAGC-3' (reverse 1), and 5'-CTAGGTGCCCTCAGTGGAGC-3' (reverse 2). PCR was carried out in $30 \mu \mathrm{L}$ reaction mixture in 200- $\mu \mathrm{L}$ PCR tubes (Axygen, Hangzhou, China), containing 50 ng genomic DNA, distilled water, 10× PCR reaction buffer (Kapa Biosystem, MA, USA), $0.6 \mu \mathrm{L}$ of each $2.5 \mathrm{mM}$ dNTPs, $10 \mu \mathrm{M}$ primer sets, and 1.5 U of Taq DNA polymerase (Kapa Biosystem). The restriction enzymes BspE1 (R108S; Enzynomics) and Sal1 (R009S; Enzynomics) were used to remove copGFP from the pCDH vector (\#CD523A-1; SBI, Palo Alto, CA, USA). The EZ-Fusion Cloning kit (EZ015M; Enzynomics) was used for cloning. Plasmid sequencing was performed using Sanger sequencing (Cosmo Genetech). To produce lentiviruses encoding each molecule $\left(\mathrm{MICA} * 002,{ }^{*} 004, * 007,{ }^{*} 008, * 009, * 010, * 011,{ }^{*} 012, * 019,{ }^{*} 027\right.$, and $\left.{ }^{*} 049\right), 5 \times 10^{6}$ HEK-293T cells/10 were seeded in T75 flasks. Twenty-four hours later, $10 \mu \mathrm{g}$ of a cloned MICA pCDH plasmid and lentivirus packaging

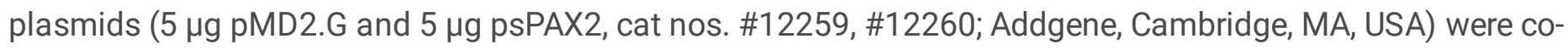


transfected into HEK-293T cells using Lipofectamine reagent (Invitrogen). At $48 \mathrm{~h}$ after transfection, lentiviral supernatants were harvested and filtered through $0.45-\mu \mathrm{M}$ filters.

\section{Generation of cell lines expressing single MICA alleles}

For transduction of each lentivirus, $5 \times 10^{5} 293 \mathrm{~T}$ cells/mL were seeded into 6-well plates. Twenty-four hours later, $500 \mu \mathrm{L}$ of lentiviral supernatant and $8 \mu \mathrm{g} / \mathrm{mL}$ of polybrene were added to $293 \mathrm{~T}$ cell cultures. At $48 \mathrm{~h}$ after transduction, the cells were cultured and analyzed using flow cytometry. Anti-MICA-PE (FAB1300P; R\&D Systems) was used. Target cells were harvested and stained with fluorescently labeled anti-human MICA antibodies for 30 min at $4^{\circ} \mathrm{C}$ in the dark. Stained cells were analyzed using FACS Canto or Fortessa flow cytometer (BD Biosciences, San Jose, CA, USA).

\section{FCM for detection of anti-MICA antibodies in sera of patients}

The recipient serum $(5 \mu \mathrm{L})$ was incubated with $20 \mu \mathrm{L}$ of the single MICA cell lines adjusted to $2 \times 10^{5} / \mathrm{mL}$ at room temperature for 30 min. Anti-human IgG Fc-FITC (F031501; DAKO, Tokyo, Japan) was used. After two washes, 2 $\mu \mathrm{L}$ of anti-human IgG (FITC-F (ab') 2 anti-human IgG, DAKO) was cross-reacted in a dark room for 30 min. After washing three times, $500 \mu \mathrm{L}$ of PBS was added to make a cell suspension, and the fluorescence response patterns were compared using FACS Canto or Fortessa.

\section{Luminex method}

MICA antibody screening was performed using LABScreen ${ }^{\text {TM }}$ MICA Single Antigen-Group 1 (One Lambda Inc.) and analyzed using a LABScan 100 flow analyzer (Luminex 100 System) according to the manufacturer's instructions.

\section{Statistical analysis}

Prism 7.0 software (GraphPad, San Diego, CA, USA) was used for data analysis and visualization. We analyzed the correlation between the FC and Luminex methods using Pearson's correlation analysis. Statistical significance was set at $P \leq 0.05$, as described in the

\section{Declarations}

\section{Acknowledgements}

This study was supported by a grant of the Korean Health Technology R\&D Project, Ministry for Health \&Welfare, Republic of Korea (HI14C3417) and the Basic Science Research Program through the National Research Foundation of Korea (NRF) funded by the Ministry of Education (2019R1I1A1A01064049)

\section{Author contributions}

Ji-Ho Jeon, In-Cheol Baek, and Tai-Gyu Kim contributed to the conception of the study; Ji-Ho Jeon was involved in data acquisition and formal analysis; In-Cheol Baek and Tai-Gyu Kim were involved in funding acquisition; Ji-Ho Jeon, In-Cheol Baek, Cheol-Hwa Hong, and Ki Hyun Park performed the investigation and methodology; Hyeyoung Lee, In-Cheol Baek, Eun-Jee Oh, and Tai-Gyu Kim were involved in project administration; Cheol-Hwa Hong, Hyeyoung Lee, and Eun-Jee Oh acquired resources; In-Cheol Baek and Tai-Gyu Kim provided supervision; Ji-Ho 
Jeon, In-Cheol Baek, and Cheol-Hwa Hong were involved in validation; Ji-Ho Jeon and Cheol-Hwa Hong performed visualization; Ji-Ho Jeon wrote the original draft, as well as reviewed and edited the manuscript.

\section{Conflict of Interest}

The authors confirm that there are no conflicts of interest.

\section{References}

1 Horton, R. et al. Gene map of the extended human MHC. Nat Rev Genet 5, 889-899, doi:10.1038/nrg1489 (2004).

2 Mizuki, N. et al. Nucleotide sequence analysis of the HLA class I region spanning the 237-kb segment around the HLA-B and -C genes. Genomics 42, 55-66, doi:10.1006/geno.1997.4708 (1997).

$3 \mathrm{Li}, \mathrm{P}$. et al. Complex structure of the activating immunoreceptor NKG2D and its MHC class I-like ligand MICA. Nat Immunol 2, 443-451, doi:10.1038/87757 (2001).

4 Groh, V. et al. Cell stress-regulated human major histocompatibility complex class I gene expressed in gastrointestinal epithelium. Proceedings of the National Academy of Sciences 93, 12445-12450, doi:10.1073/pnas.93.22.12445 (1996).

5 Groh, V., Steinle, A., Bauer, S. \& Spies, T. Recognition of stress-induced MHC molecules by intestinal epithelial gammadelta T cells. Science (New York, N.Y.) 279, 1737-1740, doi:10.1126/science.279.5357.1737 (1998).

6 Groh, V. et al. Costimulation of CD8alphabeta T cells by NKG2D via engagement by MIC induced on virusinfected cells. Nature immunology 2, 255-260, doi:10.1038/85321 (2001).

7 Schreiner, B. et al. Expression of toll-like receptors by human muscle cells in vitro and in vivo: TLR3 is highly expressed in inflammatory and HIV myopathies, mediates IL-8 release and up-regulation of NKG2D-ligands. FASEB journal : official publication of the Federation of American Societies for Experimental Biology 20, 118-120, doi:10.1096/fj.05-4342fje (2006).

8 Gasser, S., Orsulic, S., Brown, E. J. \& Raulet, D. H. The DNA damage pathway regulates innate immune system ligands of the NKG2D receptor. Nature 436, 1186-1190, doi:10.1038/nature03884 (2005).

9 Tang, K. F. et al. Decreased Dicer expression elicits DNA damage and up-regulation of MICA and MICB. The Journal of cell biology 182, 233-239, doi:10.1083/jcb.200801169 (2008).

10 Groh, V., Wu, J., Yee, C. \& Spies, T. Tumour-derived soluble MIC ligands impair expression of NKG2D and Tcell activation. Nature 419, 734-738, doi:10.1038/nature01112 (2002).

11 Groh, V. et al. Broad tumor-associated expression and recognition by tumor-derived gamma delta T cells of MICA and MICB. Proceedings of the National Academy of Sciences of the United States of America 96, 68796884, doi:10.1073/pnas.96.12.6879 (1999).

12 Terasaki, P. I. \& McClelland, J. D. MICRODROPLET ASSAY OF HUMAN SERUM CYTOTOXINS. Nature 204, 998-1000, doi:10.1038/204998b0 (1964). 
13 Terasaki, P. I. Humoral theory of transplantation. American journal of transplantation : official journal of the American Society of Transplantation and the American Society of Transplant Surgeons 3, 665-673, doi:10.1034/j.1600-6143.2003.00135.x (2003).

14 Patel, R. \& Terasaki, P. I. Significance of the Positive Crossmatch Test in Kidney Transplantation. New England Journal of Medicine 280, 735-739, doi:10.1056/nejm196904032801401 (1969).

15 Perrey, C., Brenchley, P. E., Johnson, R. W. \& Martin, S. An association between antibodies specific for endothelial cells and renal transplant failure. Transplant immunology 6, 101-106, doi:10.1016/s09663274(98)80024-5 (1998).

16 Ball, B. et al. Antibodies to vascular endothelial cells in chronic rejection of renal allografts. Transplantation proceedings 32, 353-354, doi:10.1016/s0041-1345(99)00976-8 (2000).

17 Ferry, B. L. et al. Anti-cell surface endothelial antibodies in sera from cardiac and kidney transplant recipients: association with chronic rejection. Transplant immunology 5, 17-24, doi:10.1016/s09663274(97)80021-4 (1997).

18 Zwirner, N. W., Marcos, C. Y., Mirbaha, F., Zou, Y. \& Stastny, P. Identification of MICA as a new polymorphic alloantigen recognized by antibodies in sera of organ transplant recipients. Human immunology 61, 917-924, doi:10.1016/s0198-8859(00)00162-2 (2000).

19 Williams, G. M. et al. Hyperacute Renal-Homograft Rejection in Man. New England Journal of Medicine 279, 611-618, doi:10.1056/nejm196809192791201 (1968).

20 Rebellato, L. M. et al. Clinical and anti-HLA antibody profile of nine renal transplant recipients with failed grafts: donor-specific and non-donor-specific antibody development. Clinical transplants, 241-253 (2006).

21 El-Awar, N., Lee, J. \& Terasaki, P. I. HLA antibody identification with single antigen beads compared to conventional methods. Human immunology 66, 989-997, doi:10.1016/j.humimm.2005.07.005 (2005).

22 Hancock, K. et al. False positive reactivity of recombinant, diagnostic, glycoproteins produced in High Five insect cells: effect of glycosylation. Journal of immunological methods $330,130-136$, doi:10.1016/j.jim.2007.08.002 (2008).

23 Hong, C. H., Sohn, H. J., Lee, H. J., Cho, H. I. \& Kim, T. G. Antigen Presentation by Individually Transferred HLA Class I Genes in HLA-A, HLA-B, HLA-C Null Human Cell Line Generated Using the Multiplex CRISPR-Cas9 System. Journal of immunotherapy (Hagerstown, Md. : 1997) 40, 201-210, doi:10.1097/cji.0000000000000176 (2017).

24 Mizutani, K. et al. Serial ten-year follow-up of HLA and MICA antibody production prior to kidney graft failure. American journal of transplantation : official journal of the American Society of Transplantation and the American Society of Transplant Surgeons 5, 2265-2272, doi:10.1111/j.1600-6143.2005.01016.x (2005).

25 Zou, Y., Qin, Z., Silveus, A., Fan, Y. \& Stastny, P. Polymorphisms of MICA recognized by human alloantibodies. Immunogenetics 61, 91-100, doi:10.1007/s00251-008-0344-9 (2009). 
26 Zoet, Y. M. et al. Challenging the golden standard in defining donor-specific antibodies: does the solid phase assay meet the expectations? Tissue antigens 77, 225-228, doi:10.1111/j.1399-0039.2010.01608.x (2011).

27 Zou, Y. et al. Detection of anti-MICA antibodies in patients awaiting kidney transplantation, during the posttransplant course, and in eluates from rejected kidney allografts by Luminex flow cytometry. Human immunology 67, 230-237, doi:10.1016/j.humimm.2006.02.017 (2006).

28 Baek, I. C. et al. Microarrays for high-throughput genotyping of MICA alleles using allele-specific primer extension. Tissue Antigens 82, 259-268, doi:10.1111/tan.12201 (2013).

29 Min, J. W. et al. Clinical Impact of Pre-transplant Antibodies Against Angiotensin II Type I Receptor and Major Histocompatibility Complex Class I-Related Chain A in Kidney Transplant Patients. 38, 450-457, doi:10.3343/alm.2018.38.5.450 (2018).

30 Lee, N. et al. Associations of Anti-MHC Class I-related Chain A (MICA) Antibodies with Renal Allograft Outcomes. Annals of clinical and laboratory science 50, 247-252 (2020).

\section{Tables}

Table 1. MICA allele frequencies in Koreans and MICA alleles included in cell lines expressing single MICA allele for FCA and Luminex Kit

\begin{tabular}{|c|c|c|c|}
\hline MICA alleles & Frequencies $2 \mathrm{~N}=400, \mathrm{~N}(\%)$ & FCA & Luminex \\
\hline $\mathrm{MICA}^{* *} 001$ & 0 & & $\mathrm{~V}$ \\
\hline MICA*002:01 & $42(10.5)$ & $\mathrm{V}$ & $\mathrm{V}$ \\
\hline MICA*004 & $30(7.5)$ & $\mathrm{V}$ & $\mathrm{V}$ \\
\hline $\mathrm{MICA}^{* * 005}$ & 0 & & $\mathrm{~V}$ \\
\hline $\mathrm{MICA}^{*} 006$ & $3(0.8)$ & & $\mathrm{V}$ \\
\hline MICA*007:01/02 & $13(3.3)$ & $\mathrm{V}$ & $\mathrm{V}$ \\
\hline $\mathrm{MICA}^{*} 008: 01 / 02^{¥}$ & $85(21.3)$ & $\mathrm{v}$ & \\
\hline MICA*009:01 & $17(4.3)$ & $\mathrm{V}$ & $\mathrm{V}$ \\
\hline MICA*010:01 & $69(17.3)$ & $\mathrm{V}$ & \\
\hline $\mathrm{MICA}^{*} 011$ & $9(2.3)$ & $\mathrm{V}$ & \\
\hline MICA*012:01 & $40(10)$ & $\mathrm{V}$ & $\mathrm{V}$ \\
\hline $\mathrm{MICA}^{* *} 015$ & 0 & & $\mathrm{v}$ \\
\hline $\mathrm{MICA}^{* *} 018$ & 0 & & $\mathrm{~V}$ \\
\hline MICA*017 & $2(0.5)$ & & $\mathrm{v}$ \\
\hline $\mathrm{MICA}^{*} 019$ & $11(2.8)$ & $\mathrm{V}$ & $\mathrm{V}$ \\
\hline MICA*027 & $43(10.8)$ & $\mathrm{v}$ & $\mathrm{v}$ \\
\hline $\mathrm{MICA}^{* *} 028$ & 0 & & $\mathrm{~V}$ \\
\hline $\mathrm{MICA}^{* *} 045$ & $7(1.8)$ & & \\
\hline $\mathrm{MICA}^{* *} 046$ & 0 & & $\mathrm{~V}$ \\
\hline $\mathrm{MICA}^{*} 049^{\pi}$ & $29(7.3)$ & $\mathrm{v}$ & \\
\hline
\end{tabular}


* Korean frequencies of MICA alleles have been reported in previous study ${ }^{28}$.

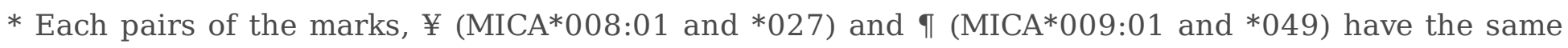
amino acid sequences of extracellular domain.

Table 2. Response patterns to each MICA alleles of FCA in 11 positive sera

\begin{tabular}{|c|c|c|c|c|c|c|c|c|c|c|c|c|c|}
\hline \multirow{2}{*}{$\begin{array}{c}\text { Sera } \\
(\mathrm{N}= \\
11)\end{array}$} & \multirow{2}{*}{$\begin{array}{c}\text { Positive } \\
\text { alleles } \\
\text { N (\%) }\end{array}$} & \multicolumn{12}{|c|}{ FCA MICA alleles $(\mathrm{N}=11, \mathrm{MFI})$} \\
\hline & & $* 002$ & $* 004$ & $* 007$ & $* 008$ & $* 009$ & $* 010$ & $* 011$ & $* 012$ & $* 019$ & $* 027$ & $* 049$ & Total \\
\hline 17498 & $8(73)$ & 149 & 1134 & 1672 & 1187 & 2879 & 484 & 41 & 1574 & 2409 & 1193 & 157 & 12879 \\
\hline 17322 & $8(73)$ & 92 & 666 & 1264 & 1094 & 1398 & 414 & 35 & 1137 & 556 & 955 & 53 & 7664 \\
\hline 16817 & $8(73)$ & 154 & 962 & 1570 & 1131 & 1874 & 356 & 38 & 1279 & 78 & 934 & 253 & 8629 \\
\hline 17649 & $7(64)$ & 518 & 251 & 436 & 210 & 549 & 164 & 392 & 326 & 1378 & 212 & 107 & 4543 \\
\hline 12862 & $6(55)$ & 745 & 433 & 432 & 344 & 287 & 232 & 11 & 142 & 19 & 378 & 148 & 3171 \\
\hline 12816 & $5(45)$ & 450 & 295 & 96 & 304 & 66 & 484 & 9 & 241 & 13 & 377 & 65 & 2400 \\
\hline 17185 & $2(18)$ & 1847 & 30 & 35 & 35 & 32 & 27 & 562 & 32 & 24 & 32 & 195 & 2851 \\
\hline 17609 & $2(18)$ & 968 & 27 & 30 & 35 & 29 & 35 & 363 & 30 & 109 & 27 & 133 & 1786 \\
\hline 12547 & $2(18)$ & 24 & 22 & 24 & 20 & 17 & 20 & 13 & 551 & 13 & 26 & 347 & 1077 \\
\hline 12610 & $1(9)$ & 26 & 28 & 32 & 106 & 28 & 86 & 11 & 80 & 13 & 50 & 458 & 918 \\
\hline 12986 & $1(9)$ & 97 & 99 & 289 & 13 & 62 & 53 & 9 & 20 & 13 & 115 & 22 & 792 \\
\hline $\begin{array}{c}\text { Positive } \\
\text { sera }\end{array}$ & N (\%) & $\begin{array}{c}5 \\
(45)\end{array}$ & $\begin{array}{c}6 \\
(55)\end{array}$ & $\begin{array}{c}6 \\
(55)\end{array}$ & $\begin{array}{c}5 \\
(45) \\
\end{array}$ & $\begin{array}{c}5 \\
(45) \\
\end{array}$ & $\begin{array}{c}4 \\
(36) \\
\end{array}$ & $\begin{array}{c}3 \\
(27)\end{array}$ & $\begin{array}{c}5 \\
(45)\end{array}$ & $\begin{array}{c}3 \\
(27)\end{array}$ & $\begin{array}{c}5 \\
(45)\end{array}$ & $\begin{array}{c}3 \\
(27)\end{array}$ & \\
\hline
\end{tabular}




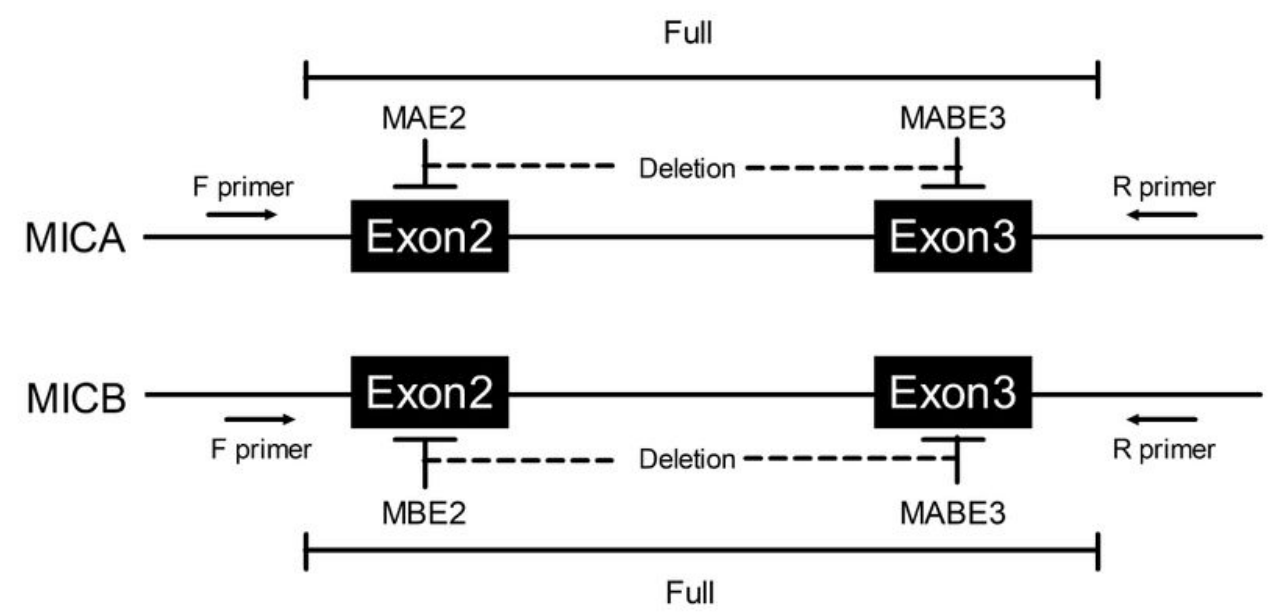

B
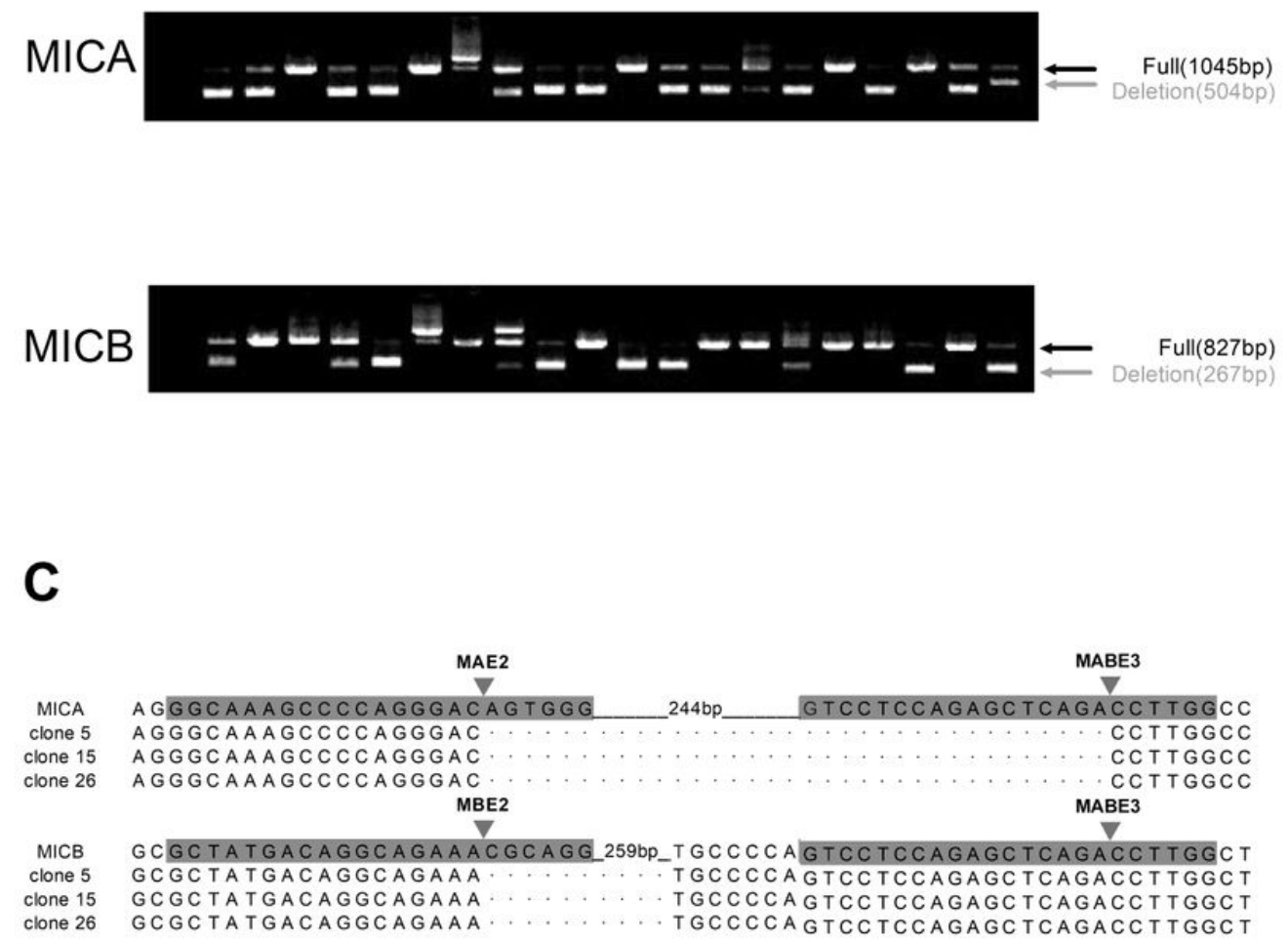

\section{Figure 1}

Additional deletion of both MICA and MICB genes on HLA class I deleted HEK-293T cell line (H1E-25) using the multiplex CRISPR/Cas9 system (A) Three guide RNA (gRNA)-Cas9 plasmids (MAE2, MBE2, and MABE3) were designed for deletions between exons 2 and 3 of each MICA and MICB gene. (B) For detection of deletion mutation, targeted PCR were performed using specific primer pairs that included exons 2 and 3 of MICA/B genes, and genotypes were analyzed using gel electrophoresis. The black arrow indicates the PCR product size of the wild-type 293T control sample. The gray arrow indicates the PCR product size of the predicted deletion between exons 2 and 3 by designed gRNAs. When the PCR product size was different from that in control cells, we designated the detectable changes in PCR products as deletions (shorter products), insertions (longer products), 
or lack of amplification. When PCR products were detected as single or double bands, they were regarded as homozygous or heterozygous. (C) Nucleotide sequences between exons 2 and 3 in the selected MICA/B null clones (clones 5, 15, and 26). The target guide RNA sequence (in gray color), cut site (inverted triangle), and removed sequence (dot) were aligned to the nucleotide sequence from wild-type HEK-293T cell line.

A

H1E-25

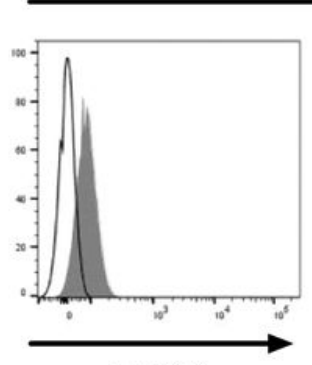

MICA
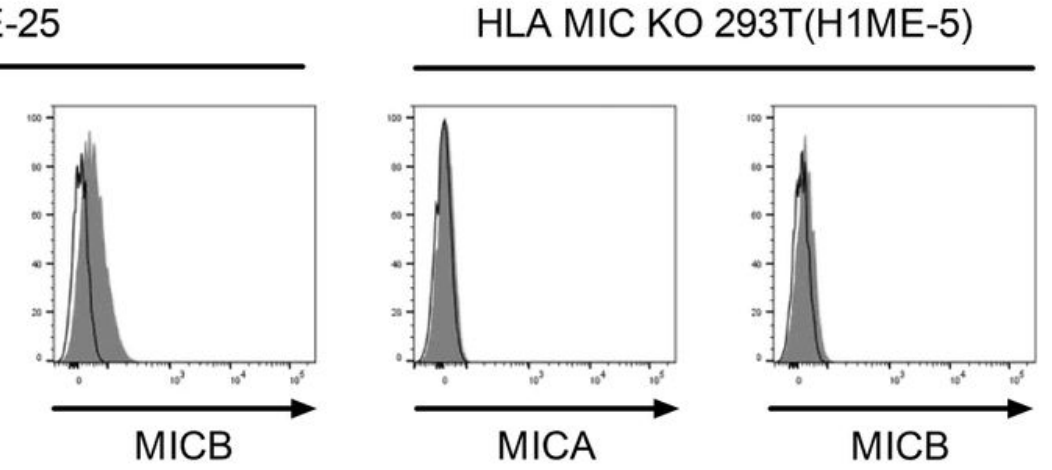

B
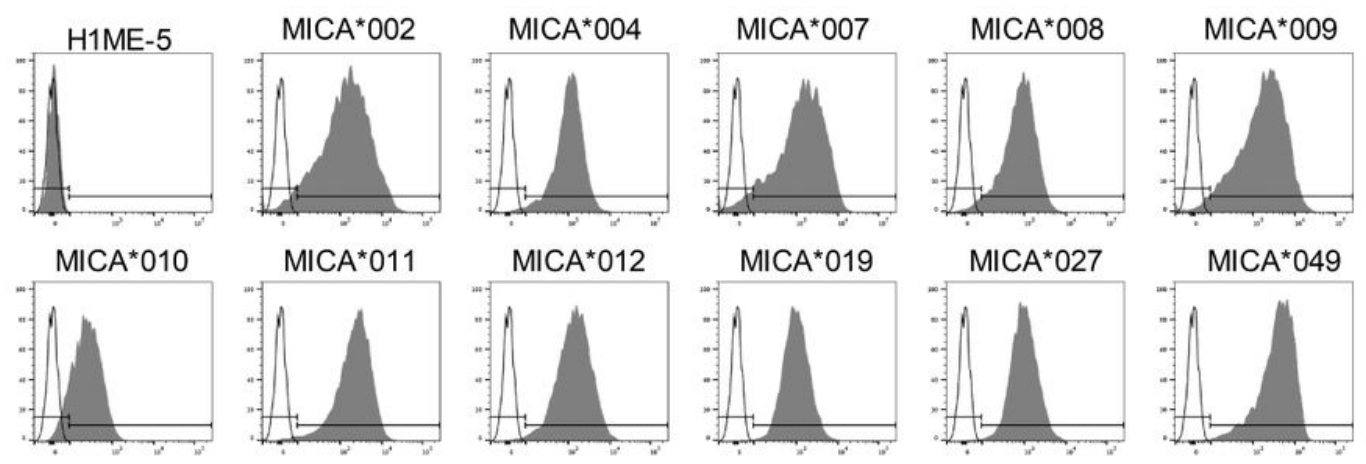

\section{C}

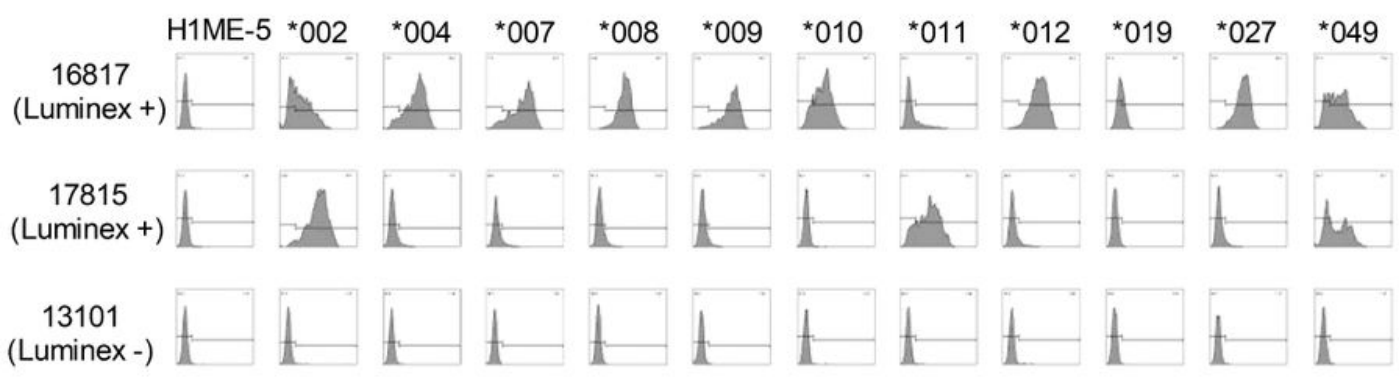

\section{Figure 2}

Establishment of cell lines expressing a single MICA allele using HEK-293T cell line (H1ME-5) from which both MICA and MICB genes have been deleted in addition to the HLA class I genes (H1E-25). (A) The expression of surface MICA and MICB molecules in H1E-25 and H1ME-5 HEK-293T cell lines. (B) Establishment of cell lines expressing single MICA allele from H1ME-5 HEK-293T cell line. Transduction and cloning using lentivirus vectors 
expressing each MICA allele genes, including MICA*002, *004, *007, *008, *009, *010, *011, *012, *019, *027, and *049. The MICA molecules expressed on the surface of selected cells were confirmed by anti-MICA PE (gray) antibody. (C) Representative response patterns of single MICA allele-expressing cell lines to the sera of two positive (16,817 and 17,815 MFI) and one negative (13,101 MFI) serum confirmed by the Luminex method.

\section{Luminex positive}
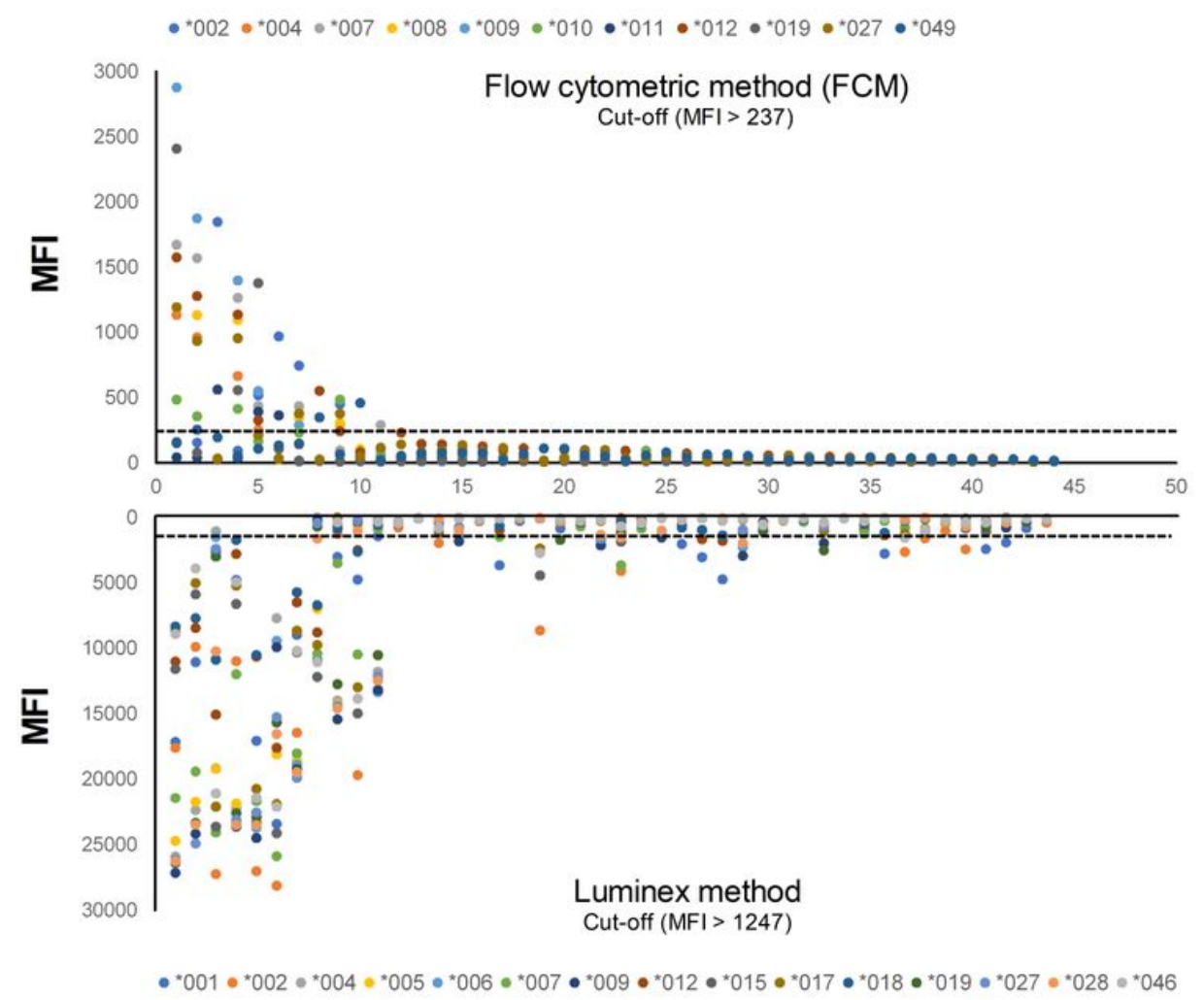

Luminex negative
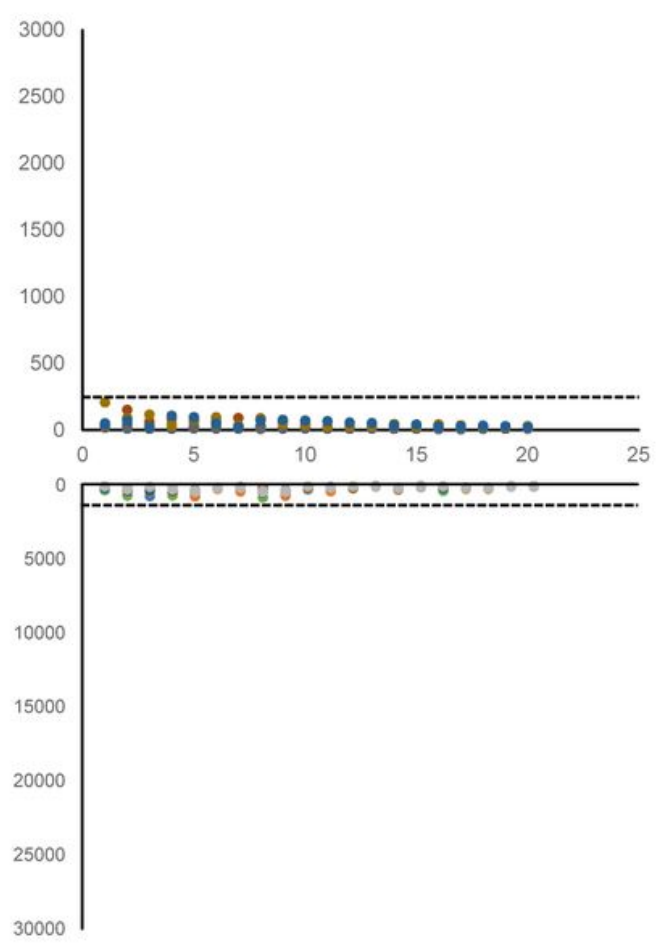

\section{Figure 3}

Comparison of response patterns to each MICA allele by flow cytometric method (FCM) and Luminex method using sera of kidney transplant patients $(n=64)$. Results were displayed in the order of sera with weak response to strong response. As for the Luminex method, 33 sera with a response of 1,247 MFI or higher were determined as positive, and, as for the FCM, 11 sera with a response of $237 \mathrm{MFI}$ or higher were determined as positive. 

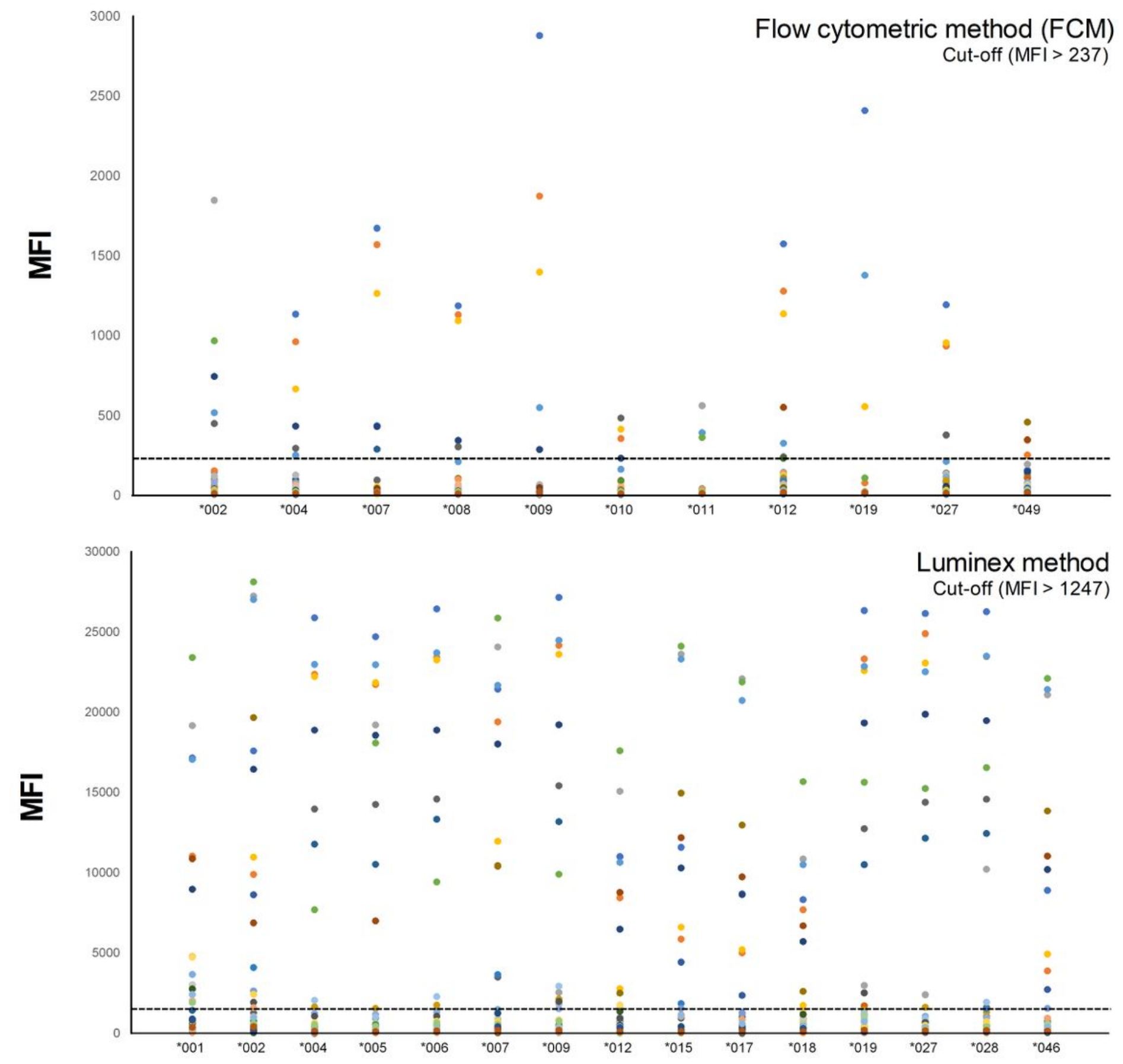

Figure 4

Response analysis for each MICA allele and flow cytometric method (FCM), Luminex method using positive sera from kidney transplant patients $(n=44)$. Both FCM and Luminex method displayed serum responses in allele order. As for Luminex method, the allele showing the strongest response was MICA*002, and, as for FCM, MICA*009 was identified as the allele showing the strongest response. 
*002

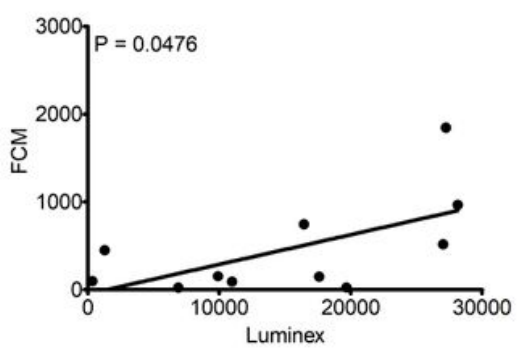

${ }^{*} 009$

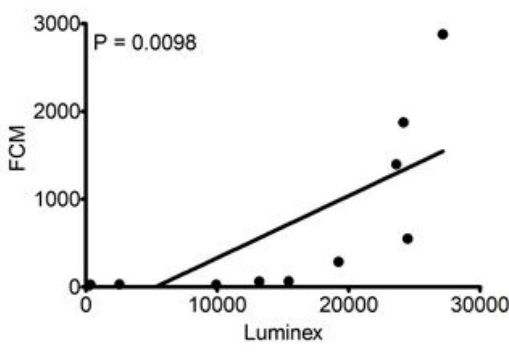

${ }^{*} 027$

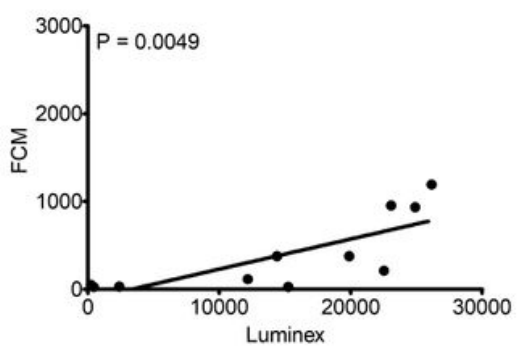

*004

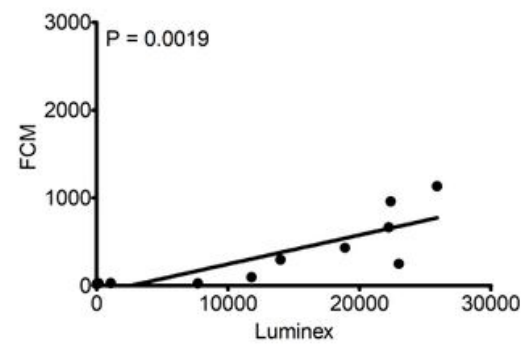

*012

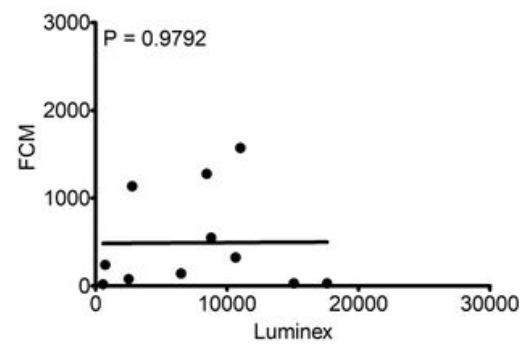

${ }^{*} 008 /{ }^{*} 027$

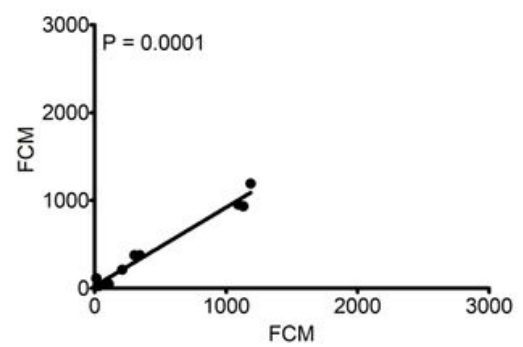

*007

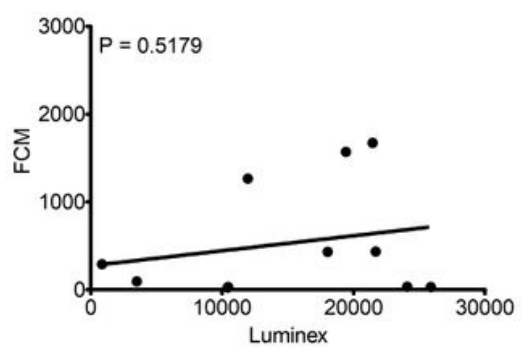

*019

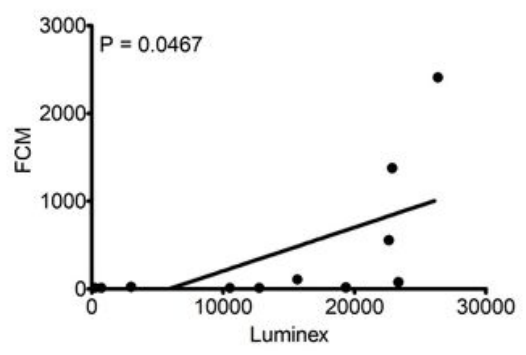

Total

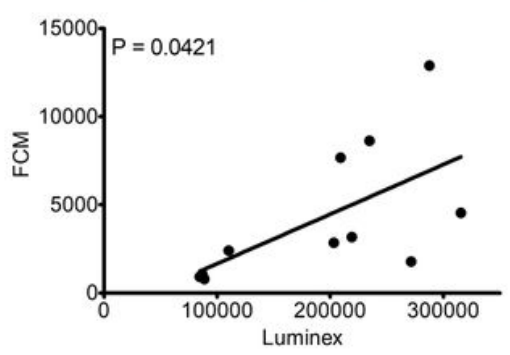

\section{Figure 5}

Correlation of total and each MICA allele response for FCM and Luminex method using 11 positive sera. The total is represented by the sum of the responses of the included alleles for each assay. Correlation was analyzed only for MICA alleles in common for both methods. 